\title{
CATASTROPHIC ANTIPHOSPHOLIPID SYNDROME (CAPS) AND THE IMPORTANCE OF SUSPECTED DIAGNOSIS AND EARLY SPECIFIC TREATMENT: CASE REPORT
}

Jacqueline Matias Moreira ${ }^{1, \star}$, Carolina Yume Arazawa1, Gilber Júnior Ferreira Gouveia ${ }^{1}$, Guilherme Maldonado Filho, Roberta Silva de Paola ${ }^{1}$, Amanda Lima de Siqueira ${ }^{1}$, Juliana Libman Luft ${ }^{1}$, Mauri Luiz Comparin ${ }^{1}$, Alex Yuzo Bueno Kanomata ${ }^{1}$, Felipe Matsushita Manzano ${ }^{1}$, Daniella Jennigs Dias $^{1}$

1.Hospital Santa Casa de Campo Grande, Campo Grande (MS), Brazil.

${ }^{\star}$ Corresponding author: jacquelinematias123@gmail.com

\section{BACKGROUND}

Catastrophic antiphospholipid syndrome (CAPS), first described in 1992, is a potentially fatal variant of antiphospholipid syndrome (PHC) and of rapid onset, occurring in $1 \%$ of patients. It is characterized by multiple vascular occlusions (usually in more than three organs or systems) in a short period of time. It is observed predominantly in women, between the second and fifth decade of life. The clinical manifestations will depend on the affected organs. In CAPS, triggering factors can be identified, such as: infections, trauma and the discontinuation of anticoagulant treatment. The diagnosis is made according to clinical and laboratory criteria. Treatment is carried out using anticoagulation, corticosteroids and plasmapheresis and / or immunoglobulin. However, with a high mortality rate even with specific treatment (ranging from $36.8 \%$ to more than $50 \%$ of cases). In this work, we report a case of CAPS that has shown good response to treatment.

\section{CASE REPORT}

Female patient, 39 years old, with a previous history of acute myocardial infarction for 8 months; in August 2020, she started pain, edema, hematomas and cyanosis in the lower limbs. In addition to also presenting an infected ulcer in the distal region of the right lower limb. Acute ischemia in the legs was diagnosed and on laboratory tests he presented with acute renal failure requiring hemodialysis. Bilateral transfemoral amputation was required, performed by the vascular surgery team. In a tomographic examination performed subsequently to investigate abdominal pain, it was observed: ischemia of the right superior mesenteric artery, splenic infarction and signs of nephropathy. In the following week, even with the use of anticoagulants at a therapeutic dose, the patient evolved with a lower level of consciousness requiring orotracheal intubation, where cranial tomography confirmed areas of acute cerebral infarction. After evaluating rheumatology and considering the possibility of CAPS, immunological exams were requested and immediate treatment with methylprednisolone (pulse therapy) associated with plasmapheresis was started; subsequently, the presence of positive lupus anticoagulants and anticardiolipin IgG was evidenced, corroborating for the diagnosis. Until the moment of producing this report, the patient is hospitalized, showing good response to clinical treatment, extubated, conscious and in gradual recovery of renal function (renal replacement therapy suspended).

\section{CONCLUSION}

The present case infers CAPS occurrence probably due to primary APS, triggered by skin infection. Due to the high rate of morbidity and mortality, early immunosuppressive therapy when CAPS is suspected, as observed in this case, seems to be a good treatment alternative. 